\title{
Factors Affecting the Outcome of Endoscopic Endonasal Transsphenoidal Surgery for Functioning Pituitary Adenomas
}

\author{
M.S.Osman, W.A.Badawy, M.M.Adawi and A.M.Nabeel \\ Neurosurgery, Dept., Faculty of Medicine, Benha Univ., Benha, Egypt \\ E-mail: dr.mhmd.saied@gmail.com
}

\begin{abstract}
Objective: Analysis of preoperative predictors for pituitary adenoma operations and their impact on results after endoscopic endonasal transsphenoidal surgery (EETSS) Methods: We carried out a prospective study of all the patients (99), who were endoscopically endonated transphenoidal at the Department of Neurosurgery, Benha University, Egypt and Wuhan Union Hospital, and China between January 2018 and December 2019. Medical data were gathered and evaluated, including information on sex, age, tumour size, hormone level, preoperative MRI brain and sella, resection scope, result (clinical and endocrinological) and complications. Results: 99 patients with PA were endoscopic transsphenoidal endonasal treatments, 52 males (52.5\%) and 47 females (47.5 percent). Average age $42.5 \pm 11.2$ years, Irregular menstrual behaviour was the highest in the study populations in 22 patients, followed by visual disturbance in 17 patients $(17.2 \%)$, acromegaly and increased intracranial pressure in 14 patients, followed by $11(11.1 \%), 8(8.1 \%)$ accidents, and $5(8.1 \%)$ cases $(11.1 \%)$, followed by headache with $11(12.1 \%)$. Conclusion: the size and scope of the tumour excision are only important postoperative determinants (hormonal, radiological \& postoperative complications).
\end{abstract}

Key words: Outcome, Pituitary adenoma, Endoscopic endonasal Transsphenoidal approach.

\section{Introduction}

Hypophysical adenomas are the third most frequent intracranial tumour, accounting between $14.4 \%$ and $22.5 \%$ in autopsy studies, but only around $10 \%$ are symptomatic [1,2].

The adenomas and null cell adenomas were most often followed by growth hormone, adrenocorticotropin and thyroid-stimulating hormone secreting adenomas, and were categorised according to their secretory activities. Hormone hyperrecretion, including hyperprolactinemia, acromegaly, Cushing's disease and hyperthyroidism, may cause patients to have welldefined clinical symptoms or with inadequate gland compression [3].

In the early post-operative period, preoperative exams of endocrinological state, field of visuals and visual acuity are also crucial to assess the response of visual and endocrine improvement following surgery. [4]

Detailed CT and MRI preoperational tests may give information on a sphenoid sinus, sellar bottom, paranasal sinuses, TB sellae, clivus, carotid artery location, optical pathways, and infundibulum. A thorough examination of lesions and their surrounding structures is essential in order to enhance surgical safety and precision [5].

Surgical treatment for non-functioning PA, GHsecretion, ACTH-secretion, gonadotropin-secretion, and TH-secretion are considered main therapies. For PA, although medical therapy is the main therapy, surgery is an option for individuals who are intolerant or drugresistant, have pituitary apoplexy, or have a development of the tumour during medical treatment [6].

Herman Schloffer initially conducted the transsphenoidal technique for the excision of pituitary adenoma more than 100 years ago. The transphenoidal technique was afterwards very interesting and many changes were made to this approach [7].

Since Schlofferin's inception in 1906, transsphenoidal surgery has progressed considerably from the use of a flashlight for vision to today's endoscopic method that makes good visualisation and considerable tumour excision possible [8].

Endoscopic chirurgy is superior than the microscopic method in the excision of sellar tumours because of greater visibility. Potential benefits include avoiding craniotomy, brain retraction and decreased neurovascular manipulation with lower morbidity. The quality of life was better than the traditional microscopic method, with regard to patient discomfort and pain perception. Operating time, hospital stay and blood loss were less endoscopic than the microscopy technique [7].

\section{Methods}

The research was performed in 99 patients with functional pituitary adenomas (fPA) at the Department of Neurosurgery of Benha University Hospitals, Egypt and Wuhan Union Hospital, China, from January 2018 to December 2019.

Functioning pituitary adenomas were identified based on clinical properties, hormone tests and confirmation of CT and/or MRI pituitary adenomas.

Sized tumours were categorised in the categories of microadenoma ( $<10 \mathrm{~mm})$, macroadenoma (al-10 mm) and gigantic adenoma $(>4 \mathrm{~cm})$ in their greatest diameter.

All patients had endoscopic transsphenoidal transnasal surgery, followed by a tissue investigation. Further preoperative data includes age and sex.

Patients were assessed on a clinical, hormonal and radiological basis based on improvement. On the first post-operative day, a follow-up was performed 3 months later and up to 36 months following the procedure to evaluate treatment modality outcomes.

\subsection{Endocrinological evaluation}

Hormonal assays preoperative (basal) and postoperative including; anterior pituitary functions were assessed in all patients. The following hormonal dosages were obtained: serum prolactin (PRL), growth hormone 
(GH), insulin-like growth factor (IGF1), thyroid stimulating hormone (TSH), adrenocorticotropic hormone $(\mathrm{ACTH})$, luteinizing hormone $(\mathrm{LH})$, follicle stimulating hormone (FSH), free thyroxine (FT4), free triiodothyronine (FT3), serum cortisol and urinary free cortisol.

\subsection{Surgical technique}

The patient was put in the supine position under general anaesthesia without strict routine immobilisation of the head. Both the surgeon and the helper were on the right side of the patient. After the nasal and oral cavities had been rigorously sterilised, pieces of cotton soaking with adrenaline were used to constrict blood vessels and expand the nasal cavity. Operation is typically conducted using a $0^{\circ}$ rigid endoscope binostril route. The lower turbinate and nasal septum are visible after the endoscope is placed into the nasal septum. Then a medium turbinate was lateralized for the surgical corridor and a $1.0-1.5 \mathrm{~cm}$ above the sphenoethmoid recess could be observed in the sphenoid sinus ostium, an important anatomy marker. To connect bilateral nasal cavities and expand the work space the back nasal septum was removed and the anterior wall and partial septum of the sphenoid sinus combined were removed. The dura was opened with a cross-section and the tumour was removed piece by piece using a curette, tweezers, and aspirator after removing the remaining septal of the sphenoid sinus. After the tumour had been removed, we utilised gelfoam to seal the surgical cavity and a neuropatch was inserted into the dural defect. When a postoperative cerebrospinal fluid (CSF) leak is anticipated, two pieces of fascia lata attached by the fibrin glue are used to scale the surgical hole. A lumbar catheter is then inserted until the third postoperative day for CSF draining. The mucosal incision is subsequently closed and the two narrows pad 24 hours.

\section{Results}

Our patient population in this study included 99 patients, 52 males $(52.5 \%)$ and 47 females (47.5\%). The mean age was $42.5 \pm 11$.2years, with a range of 15 66.

Irregular menstruation was the most common presenting symptoms among the study population in 22 patients $(22.2 \%)$, followed visual disturbance in 17 patients $(17.2 \%)$, acromegaly and increased intracranial pressure (inform of combination of headache, nausea, vomiting \& visual disturbance) were in third place with each one 14 patients $(14.1 \%)$, then headache with 11 patients $(11.1 \%), 8$ cases $(8.1 \%)$ were accidently Preoperative imaging discovered, 5 cases $(5.1 \%)$ had Cushing disease, also 5 cases $(5.1 \%)$ had sexual dysfunction, finally 3 cases $(3 \%)$ were recurrent pituitary adenomas. 80 cases $(80.8 \%)$ were marcroadenoma $(>1 \mathrm{~cm})$, while 8 cases $(8.1 \%)$ were microadenoma $(<1 \mathrm{~cm})$ and 11 cases $(11.1 \%)$ were giant adenomas $(>4 \mathrm{~cm})$. In our study on functioning pituitary adenomas; 55 patients (55.6\%) were prolactiomas, while 17 patients $(17.2 \%)$ were $\mathrm{GH}$ secreting adenomas, 11 patients (11.1\%) were Gonadotropin hormone - secreting adenomas, 6 patients (6.1\%) were ACTH - secreting adenomas, 2 patients (2\%) were thyrotropin (TSH) -secreting adenomas and 8 patients $(8.1 \%)$ were multiple hormones secreting adenomas. 79 patients $(79.8 \%)$ underwent gross total resection of the tumor, while 20 patients (20.2\%) underwent subtotal resection of the tumor. mean of KI 67 labeling index in immunohistochemistry was $2.5 \pm 2.2 \%$. In our study; 93 patients $(93.9 \%)$ were clinically improved while 6 patients $(6.1 \%)$ were not improved. Also, we found that 96 patients $(97 \%)$ were improved hormonally while 3 patients $(3 \%)$ were not improved. 64 patients $(64.6 \%)$ passed without complications but 35 patients $(35.4 \%)$ had complication inform of 14 patients (14.1\%) had hypopituitarism, 5 patients $(5.1 \%)$ had transient diabetes insipidus, 5 patients $(5.1 \%)$ had csf leak, 4 patients $(4 \%)$ had hyponatremia, 4 patients $(4 \%)$ developed anosmia, 2 patients $(2 \%)$ had epistaxis and one patient $(1 \%)$ died due to acute hydrocehallus and cerebral haemorrhage, In our study after neuroimaging (MRI) follow up; 79 patients $(79.8 \%)$ were totally removed and 20 patients $(20.2 \%)$ had residual of the tumor.

Case 62

Male patient 41 years old with Pituitary macroadenoma, complaint of acromegaly and visual disturbance. Preoperative endocrine evaluation: High GH, High IGF-1, otherwise normal, Preoperative MRI: Sellar and suprasellar mass with left cavernous sinus invasion, extend superioly compressing optic chiasm and 3rd ventricle, Size of the adenoma: Macroadenoma, Hormone typing: GH adenoma, Surgical approach: Endoscopic endonasal trans-sphenoidal approach. Extent of resection: subtotal resection, Pathology: Pituitary adenoma. Immunohistochemistry: Syn + , ACTH -, GH +, PRL: partial +, FSH -, LH -, TSH -, Ki67: about 5\%. Fig. (1) Follow-up time: 7 month. Postoperative complications: Low cortisol level. Clinical follow-up: improved. Hormonal follow up: Decreased level of GH \& IGF-1 but still above normal, low cortisol level below normal, otherwise normal. MRI follow-up: residual encasing left cavernous sinus for gamma knife Fig. (2).

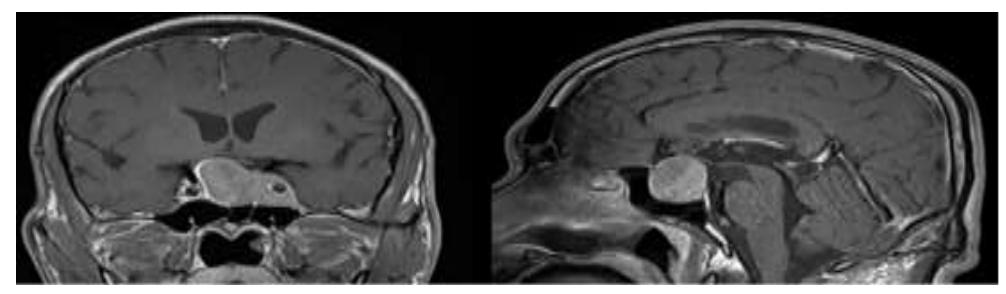

Fig. (1) Pre-operative MRI: T1 with contrast coronal \& sagittal views. 
Postoperative imaging ( $3 \mathrm{~m}$. later)

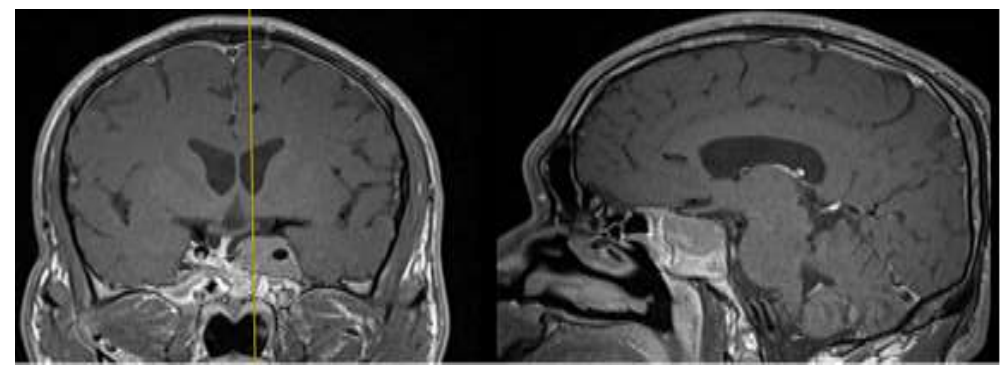

Fig. (2) Post-operative MRI: T1 with contrast coronal \& sagittal views

\section{Preoperative imaging}

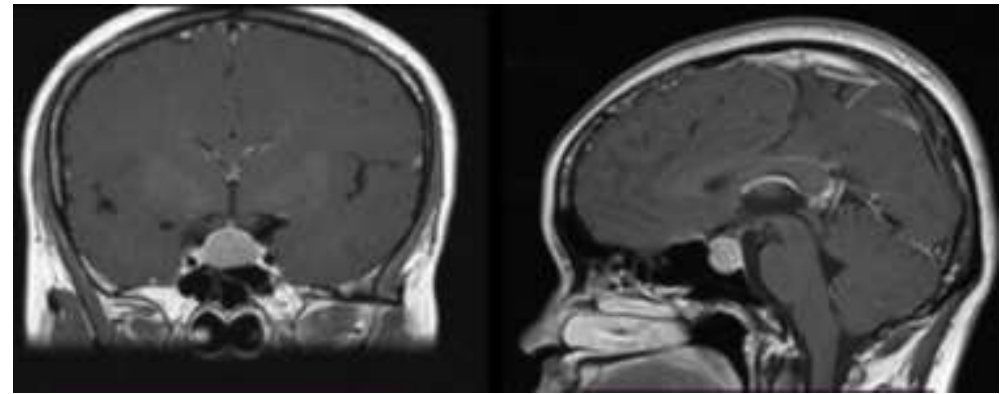

Fig. (3) Pre-operative MRI: T1 with contrast coronal \& sagittal views

\section{Postoperative images (3 months later)}

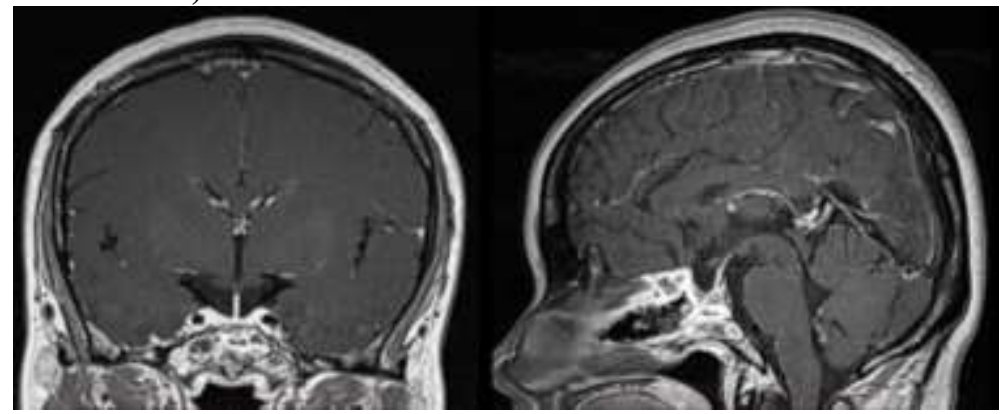

Fig (4) Post-operative MRI: T1 with contrast coronal \& sagittal views

\section{Case 57}

Female patient 36 y had Pituitary macroadenoma, complaint with Irregular menstruation, both fundus ex. \& visual field normal, Preoperative endocrine evaluation: High prolactin, otherwise normal, Preoperative MRI: Pituitary macroadenoma with no mass effect upon optic chiasm nor 3rd ventricle and no suprasellar extension or invasion to the cavernous sinus. Size of the adenoma: macroadenoma, Hormonetyping: prolactinoma, surgical approach: Endoscopic endonasal trans-sphenoidal approach, Extent of resection: Gross total resection, pathology: pituitary adenoma, Immunohistochemistry: Syn+ACTH -,GH -, PRL + , FSH +, LH-, TSH focal weak + , ki67:2\%, Fig. (3) follow-up time: 7 month, postoperative complications: none, Clinical follow-up: totally improved, Hormonal follow up: all normal, MRI follow-up: No evidence of tumor residual or recurrence after 7 month from surgery Fig. (4).

\section{Discussion}

This research was performed at the Department of Neurosurgery at University Hospitals in Benha; Egypt and Wuhan Union Hospital in China in ninety-nine (99) patients with functional pituitary adenomas (FPA) between January 2018 and December 2019.

We enrolled 99 patients, 52 men (52.5 percent) and 47 women in our research (47.5 percent ). We have also discovered that the age of the patients examined varied from 15 to 66 years, with a mean age of $42.5 \pm 11.2$ years.

In the 2017 series Jiang et al., which included 171 patients, 101 men (59.1 percent) and 70 women (40.9 percent) were sampled, with an average age of $47.4 \pm$ 12.8 years and a range of 15-71 years.[6] In the Day et al series published during 2016, including 101 patients, mean diagnosed PA age was 46.39 (18.2) years. The Gondim et al. series published in 2010 comprised 99 men (53.8 percent) and 85 females (46.2 percent), 
respectively 44.7 percent (102 patients) and 55.3 percent (126 patients) of them, respectively. The average age of the population examined was $42,51 \pm 15,25$ years $(13-79$ years) [5].

In our study the most common symptoms in the study population in 22 patients were irregular menstrual conditions, followed by visual disorder (17.2 percent), acromegaly and increased intra-crane pressure (headaches, nausea, vomiting and visual disorder) in 17 patients, with each 14 patients (14.1 percent), then headaches with a combination of headache, nausea and vision disorder.

Headache (65 patients, 38.01\%), visual impairment (visual loss, diplopia and field of vision defaults of 64 patients, $37.43 \%$ ), menstruation problem or lactation (27 patients, $15.79 \%$ ) and acromegaly were major symptoms in the 2017 dataset, comprising 171 individuals $(9$ patients, 5.26 percent ). Moreover, 18 patients (10.53 percent) were accidentally identified without any clear symptoms.[10]

In the 2014 Bodhinayake et al. series, 64 patients, headaches and visual impairments were the most frequent complaints at first assessment in $43.8 \%$ and $39.1 \%$, respectively. Amenorrhea: 10 patients (15.6 percent), 11 incidentals (17.2 percent), 13 patients with galactorrhea (20.3 percent), 5 patients with libido reduced (7.8 percent), 6 patients with diplopia (9.4 percent), 3 with acromegaly, 4.7 percent, 1 with apoplexy, 1 with seizure (1.6 percent), patients. [8]

In our research, $80(80.8 \%)$ instances were marcroadenoma $(>1 \mathrm{~cm})$, whereas $8(8.1 \%)$ were microadenoma $(<1 \mathrm{~cm})$, and $11(11.1 \%)$ were huge adenomas.

In Jiang et al, the 2017 series included 171 patients, 133 macroadenomas $(77.78 \%), 20$ microadenomas (11.70\%) and 18 Giant adenomas (10.52\%)[6].

In Chen et al. series released in 2011, 289 individuals $(75.1 \%), 30(7.8 \%)$ were microadenomas, and $66(17.1 \%)$ were large adenomas, totaling 385 patients $(75.1 \%)$.

In the 2012 series Marić et al. reported, 117 patients, 63 macroadenomas $(53.8 \%)$ and 54 patients (46.2\%) microadenomas (12\%).[12]

55 patients $(55.6 \%)$ were prolactiomas, 17 patients (17.2\%) were adenomas-related GH-secreting, 11 patients $(11.1 \%)$ were adenomas-related Gonadotropinsecreting, 6 patients $(6,1 \%)$ were ACTH-secreting adenomas, 2 patients $(2 \%)$, were thyrotropin-secreting adenomas and 8 patients $(8,1 \%)$ were multi-adenomas.

In the 2006 published series of Daly et al., which included 68 patients, $45(66 \%)$ were prolactioms, whereas 9 (13\%) were $\mathrm{GH}$ - secreting adenomas, 4 (6\%), ACTH - secreting adenomas.13 The Fontana et al. series, published in 2009, comprises 44 patients, 25 $(56 \%)$ were prolactioms, and $4(9 \%)$ were GH secreting adenomas.

In our study: $79(79.8 \%)$ patients had gross complete tumour excision, whereas $20(20.2 \%)$ were subtotal tumour resection patients. Three patients had a second endoscopic or craniotomy surgery, and 18 had post-operative therapy with gamma knife.

In the 2017 study of Jiang et al., comprising 171 patients, 126 patients $(73.68 \%)$ had gross total resection (GTR), 4 patients were subjected to second microscope or endoscopy surgery and eleven were subjected to postoperative gamma clothing therapy.

The mean KI 67 labelling index in our research was $2.5 \pm 2.2 \%$, ranging from $0.1 \%$ to $10 \%$.

The average Ki-67 labelling index was 0.81 percent in Paek et al. series reported in 2005, comprising 44 patients and varied from 0.1 to 4 percent. 16 The mean Ki-67 LI was 2,4 60,3 percent for the 101 patients reported in the 2000 Losa et al. series, comprising 101 individuals, of 0,0 to 23,0 percent. [17]

The average length of our research was 12.9 months (range 3-36 months).

In the 2014 series of 64 of Bodhinayake et al., the average follow-up period for this research was 24.3 months (median 23.1, range 4.0-62,5).[8]

In our research, 93 patients $(93.9 \%)$ were better clinically whereas six $(6.1 \%)$ were not improved. We also observed that 96 patients $(97 \%)$ had improved hormonally, whereas 3 patients $(3 \%)$ had not improved.

In Jiang et al. 2017, comprising 171 patients, the symptoms of 144 patients (95.36 percent) improved substantially, or in 151 individuals, vanished (20 patients did not have any obvious symptoms). [6]

6 All patients were examined with relative pituitary hormone within 1 week of surgery. In 40 patients $(23,39$ percent), however, only 6 of them needed long-term hormone replacement treatment, while other patients were soon recovered from normal hormone levels. [6]

In Chen et al. series published in 2011, 385 of these discharged patients were better, including visual disturbance in 215 (87.6 percent) preoperatively complaining patients.[11]

11 Follow-up following neuroimaging (MRI) in our research; 79 (79.8 per cent) patients were completely eliminated and 20 (20.2 per cent) patients had tumour rest. Three patients had a second endoscopic or craniotomy surgery, and 18 had post-operative therapy with gamma knife.

In the 2014 Bodhinayake et al. series, which included 64 patients, 20 patients $(31.3 \%)$ had residual tumour, and $44(68.7 \%)$ had GTR without tumour remaining. [8]

The improved MRI scans were first conducted around four months following surgery and afterwards in the internal year of the Chen et al. Series published in 2011, comprising 385 patients. The rest of the (noncomplete) tumour was verified in 79 patients $(20.5 \%)$ after first surgery, and the TSS was done a second time (8 instances) if the remaining tumour was crushed above the sella turcica or a reasonably short gap between the optic chiasm and the tumour had been compressed. The tumour was otherwise sent for postoperative radiation with a gamma knife (35 cases). [11]

In our study 64 patients $(64.6 \%)$ had hypopituitarism in 14 patients, but 35 patients $(35.4 \%)$ 
had complication reporting in 5 patients $(5.1 \%)$, insipid transient diabetes, 5 patients $(5.1 \%)$ had csf leak, 4 patients (4\%) had pituitaries, 4 patients $(4 \%)$ had anosmia, 2 patients $(2 \%)$ had epistaxy

Surgeonic complications have been reported in 55 patients, including hypopituitarism (6 patients, 3.51\%), post-operative CSF leak (3 patients, $1.75 \%$ ), temporary diabetes insipidus (14 patients, 8.19\%), hyposmia or anosmia (24 patients, 14.04\%), cerebral blood (4 patients, 2.34\%) in Jiang et al. series published in 2017, including 171 patients (4 patients, 2.34 percent ). Hyposmia or anosmia, which progressively came back in most patients, was the most frequent postoperative consequence. [11]

126 trans-sphenoidal surgeries were conducted in the research by Sudhakar et al. in 108 individuals with pituitary pathology. The most frequent was diabetes insipidus (DI) (23 percent ). Other significant problems included: $22 \%$ postoperative hypopituitarism, $13 \% \mathrm{CSF}$ leak, $5.5 \%$ meningitis and 2,4\% visual impairment; $1 \%$ haematoma; and a $0.8 \%$ death rate of 30 days. Subdural haematoma, epistaxis, SIADH and hydrocephalus were further problems. [18]

The tumour size substantially affected surgical success, the postoperative remission (hormonal and radiological) was obtained in $83,8 \%(98 / 117)$ of patients in Marić et al. series reported in 2012: 69,8\% (44/63), and $100 \%$ with microadenomas (54/54).

12 The size of the tumour has greatly impacted endocrinological problems. Microadenomas patients had substantially reduced incidence of complication. [12]

In 20 patients treated by endonasal endoscopy, Jain et al. examined the connection between the size of hypophysical adenomas and the result. They found that the volume of the tumour less than $5 \mathrm{ml}(\mathrm{P}<0.05)$ and no parasellar or suprasellar extension are favourable for complete deletion. [19]

There was no significant link between the hormonal type of the patients examined and hormonal improvement, post-operative complication and follow-up to MRI (presence of residual or not).

There were also no significant variations between complication rates and type of tumour in the Maric et al. dataset published in 2012 comprising 117 people; type of tumour did not substantially impact endocrinological or radiological surgical outcomes $(\mathrm{P}=0,073)$ [12]

In our research the relationship between the amount of tumour excision and hormone improvement, postoperative complication and the existence or not of residual mass was statistically significant.

$100 \%$ of patients with complete tumour resection exhibited a hormone improvement and no MRI followup of residual mass $(\mathrm{p}=0.000)$. And $69.6 \%$ of them had no postoperative problem $(\mathrm{p}=0.04)$.

In our research there was no significant connection between Ki-67 Labeling Index and hormonal improvement, morbidity and neuroimaging after surgery (presence of residual or not)

There was no significant connection between the proliferation and the hormonal states of the adenomas in
Paek et al. series reported in 2005, comprising 44 individuals.

The Ki-67 index does not seem to offer independent information on the identification of tumour recurrences. Although studies of the long-term follow-up Ki-67 index are sparse, the index seems to offer important predictive information. The high Ki-67 index may indicate a thorough clinical and radiological follow-up to identify the proper features and recurrence of pituitary adenomas and thus a suitable treatment approach [16].

In the Losa et al. research of patients with PAs that are not chosen, it has been shown that the Ki-67 LI is not related with clinical, hormonal or residual features in the follow-up [17].

\section{Conclusion}

Tumor size and extent of resection are only significant factors for postoperative follow up (hormonal, radiological \& postoperative complications).

\section{References}

[1] A.Rudnik, T.Zawadzki, B.Gałuszka-Ignasiak, P.Bazowski, I.Duda, M.Wojtacha. Endoscopic transsphenoidal treatment in recurrent and residual pituitary adenomas-first experience. Minim Invasive Neurosurg.vol.49,pp.10-14,2006.

[2] HM.Negm, R.Al-Mahfoudh, M.Pai. Reoperative endoscopic endonasal surgery for residual or recurrent pituitary adenomas. J Neurosurg..vol.127(2),pp.397-408. doi:10.3171/2016.8.JNS152709,2017.

[3] KI. Lampropoulos, G.Samonis, P.Nomikos Factors influencing the outcome of microsurgical transsphenoidal surgery for pituitary adenomas: a study on 184 patients. Hormones (Athens).vol.12(2),pp.254-264,2013.

[4] I.Anik, Y.Anik, Koc K. Evaluation of early visual recovery in pituitary macroadenomas after endoscopic endonasal transphenoidal surgery: Quantitative assessment with diffusion tensor imaging (DTI). Acta Neurochir (Wien).vol.153(4),pp.831-842. doi:10.1007/s00701-011-0942-4,2011.

[5] Y.Yadav, S.Sachdev, V.Parihar, H.Namdev, P.Bhatele Endoscopic endonasal trans-sphenoid surgery of pituitary adenoma. J Neurosci Rural Pract.vol.3(3),pp.328-337. doi:10.4103/0976$3147.102615,2012$.

[6] X. Jiang, Z. Liu, X. Huang, H. Wang, H.Zhao Extended endoscopic endonasal approach for pituitary adenoma: A single-center experience of 171 patients. Chinese Neurosurg J. Jun .vol.6;3(1),pp.1-8,2017.

[7] CP.Hofstetter, BJ.Shin, L.Mubita Endoscopic endonasal transsphenoidal surgery for functional pituitary adenomas. Neurosurg Focus.vol.30(4),pp.E10. doi:10.3171/2011.1.FOCUS10317,2011. 
[8] I. Bodhinayake, M.Ottenhausen, MA.Mooney. Results and risk factors for recurrence following endoscopic endonasal transsphenoidal surgery for pituitary adenoma. Clin Neurol Neurosurg.vol.119,pp.75-79. doi:10.1016/j.clineuro.2014.01.020,2014.

[9] PF.Day, MG.Loto, M.Glerean. Incidence and prevalence of clinically relevant pituitary adenomas: retrospective cohort study in a Health Management Organization in Buenos Aires, Argentina. Arch Endocrinol Metab.vol.60,pp.554561. doi:10.1590/2359-3997000000195,2016.

[10] JA.Gondim, M.Schops, JP.de Almeida. Endoscopic endonasal transsphenoidal surgery: surgical results of 228 pituitary adenomas treated in a pituitary center. Pituitary.vol.13(1),pp.68-77. doi:10.1007/s11102-009-0195-x,2010.

[11] L.Chen, WL.White, RF.Spetzler, B.Xu A prospective study of nonfunctioning pituitary adenomas: presentation, management, and clinical outcome. J Neurooncol.vol.102(1),pp.129-138. doi:10.1007/s11060-010-0302-x,2011.

[12] A.Marić, I.Kruljac, V.Čerina, HI.Pećina, P.Šulentić, M.Vrkljan Endocrinological outcomes of pure endoscopic transsphenoidal surgery: a Croatian Referral Pituitary Center experience. Croat Med J.vol.53(3),pp.224-233. doi:10.3325/cmj.2012.53.224,2012.

[13] AF.Daly, M.Rixhon, C.Adam, A.Dempegioti, MA.Tichomirowa, A..Beckers High prevalence of pituitary adenomas: a cross-sectional study in the province of Liege, Belgium. J Clin Endocrinol Metab.vol.91(12),pp.4769-4775. doi:10.1210/jc,2006.

[14] E.Fontana,R.Gaillard Epidémiologie des adénomes hypophysaires: étude dans une agglomération urbaine de Suisse [Epidemiology of pituitary adenoma: results of the first Swiss study]. Rev Med Suisse.vol.5(223),pp.2172-2174,2009.

[15] A.Raappana, J.Koivukangas, T.Ebeling, T.Pirilä Incidence of pituitary adenomas in Northern Finland in 1992-2007. J Clin Endocrinol Metab.vol.95(9),pp.4268-4275. doi:10.1210/jc.2010-0537,2010.

[16] KI.Paek, SH.Kim, SH.Song. Clinical significance of Ki-67 labeling index in pituitary macroadenoma. J Korean Med Sci.vol.20(3),pp.489-494. doi:10.3346/jkms.2005.20.3.489,2005.

[17] M.Losa, A.Franzin, F.Mangili. Proliferation index of nonfunctioning pituitary adenomas: correlations with clinical characteristics and longterm follow-up results. Neurosurgery.vol.47(6),pp.1313-1319,2000.

[18] N.Sudhakar, A.Ray, JA.Vafidis Complications after trans-sphenoidal surgery: our experience and a review of the literature. $\mathrm{Br} J$ Neurosurg.vol.18(5),pp.507-512. doi:10.1080/02688690400012459a,2004.
[19] AK.Jain, AK.Gupta, A.Pathak, A.Bhansali, JR.Bapuraj Endonasal transsphenoidal pituitary surgery: is tumor volume a key factor in determining outcome?. Am J Otolaryngol.vol. 29(1),pp.48-50,2008. 